\title{
Thrombocytopénie immunologique sévère liée à l'infection VIH : intérêt des immunoglobulines polyvalentes intraveineuses avant chirurgie buccale. À propos d'un cas.
}

\section{Severe immune thrombocytopenia associated with VIH infection: usefulness of intraveinous polyvalent immunoglobulins before oral surgery. Case report.}

CÉDRIC MAUPRIVEZ*, VINCENT FURON* ${ }^{\star}$ THOMAS DUFOUR*, CHRISTINE ROUGER**, BENOÎT LEFÈVRE*

\section{RÉSUMÉ}

La thrombocytopénie est une manifestation biologique relativement fréquente de l'infection par le VIH.

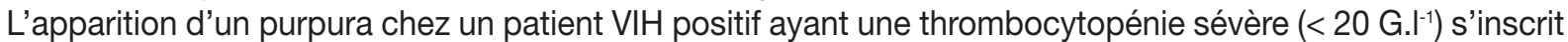
le plus souvent dans le cadre d'un purpura thrombopénique immunologique (PTI). Le mécanisme évoqué est une hyperdestruction splénique des plaquettes du fait de l'existence d'autoanticorps antiplaquettes. Un cas d'utilisation d'immunoglobulines humaines polyvalentes par voie intraveineuse (IgIV) dans le contrôle du risque hémorragique lié au PTI en vue d'extractions dentaires est rapporté. (Med Buccale Chir Buccale 2004; 10: 71-76)

mots clés : Thrombocypénie, Infection VIH, Immunoglobulines polyvalentes intraveineuses, Chirurgie buccale.

médecine

buccale

chirurgie

buccale

VOL. $10, \mathrm{~N}^{\circ} 2$ 2004

page 71

\section{SUMMARY}

Thrombocytopenia is a relatively common manifestation of HIV infection. Emergence of purpura in HIV-infected patients with severe thrombocytopenia $\left(<20 \mathrm{G}^{-1} \mathrm{I}^{-1}\right)$ is more often associated with an immune thrombocytopenic purpura (ITP). The mechanism met is the spleen platelets destruction because of antiplatelets antibodies. A case of intraveinous human polyvalent immunoglobulins (IgIV) for the bleeding risk control associated with ITP in dental extractions is reported. (Med Buccale Chir Buccale 2004; 10: 71-76)

key words : Thrombocytopenia, HIV Infection, Intravenous polyvalent immunoglobulins, Oral surgery.

\footnotetext{
* Service d'odontologie, Unité de pathologie et chirurgie buccale, CHU Reims

** Service de Médecine Interne et Maladies Infectieuses, CHU Reims
}

Demande de tirés à part :

Cédric Mauprivez - Service d'odontologie - Unité Pathologie et Chirurgie Buccale - CHU Reims Hôpital Maison Blanche

45 rue Cognacq Jay - 51092 Reims Cedex 
médecine

buccale

chirurgie

buccale

VOL. $10, \mathrm{~N}^{\circ} 2$ 2004

page 72
La thrombocytopénie est l'anomalie de l'hémostase la plus fréquemment rapportée lors de l'infection VIH [1-7]. Sa prévalence va de $9,2 \%$ à $21,2 \%$ selon les études [2]. Elle peut être observée quel que soit le mode de contamination et à tous les stades de la maladie [5-7]. Sa fréquence augmente avec la baisse des $\mathrm{CD} 4^{+}$et le passage au stade SIDA [2,5]. En revanche, le diagnostic d'une thrombocytopénie n'est pas un élément péjoratif indiquant une évolution plus rapide vers le stade SIDA [2,5,7].

La thrombocytopénie est le plus souvent directement liée à l'infection $\mathrm{VIH}$; elle constitue un des symptômes biologiques de la maladie [1]. Les autres causes de thrombocytopénie chez les patients infectés par le VIH sont : une toxicité médicamenteuse (antiviraux, antibiotiques...), une atteinte de la moelle osseuse par un lymphome, une infection mycobactérienne ou une infection fongique (cryptococosse, histoplasmose) $[1,3,7]$.

La physiopathologie de la thrombocytopénie due à l'infection VIH est complexe et probablement multifactorielle. Elle peut être d'origine périphérique par destruction immunologique ou centrale par défaut de production $[2,5,7]$.

La destruction périphérique des plaquettes est due à la présence dans le sérum des patients d'autoanticorps dirigés spécifiquement contre certains déterminants antigéniques de la membrane des plaquettes [2,5]. L'existence de ces autoanticorps antiplaquettes résulterait d'une réactivité humorale croisée entre des constituants viraux et plaquettaires, en particulier entre la GP 120 et la GP IIb/IIla [4]. La fixation de ces autoanticorps sur les plaquettes permet la destruction de ces dernières par les phagocytes mononucléés en particulier spléniques. Cette thrombocytopénie d'origine immunologique est généralement sévère et d'apparition brutale. La durée de vie des plaquettes dans le sang périphérique est considérablement réduite, elle est en général inférieure à 24 heures, avec le plus souvent la mise en évidence d'une séquestration splénique [5].

Chez les malades présentant une infection plus ancienne et plus évoluée, une insuffisance de production médullaire s'ajoute à l'hyperdestruc- tion périphérique [2,5,7]. Cette origine centrale, par déficit de la production plaquettaire, serait dû à un envahissement médullaire et à une toxicité directe du VIH sur les mégacaryocytes [1,2,5]. Elle doit être évoquée en présence d'une pantocytopénie; par ailleurs elle est partiellement réversible avec un traitement antirétroviral par AZT [2,5].

La thrombocytopénie liée au VIH est le plus souvent asymptomatique [6,10]. Le diagnostic est porté à l'occasion d'hémogrammes pratiqués de façon systématique. Lorsque la thrombocytopénie est sévère avec un taux de plaquettes en-dessous de $20 \mathrm{G} \mathrm{l}^{-1}$, des signes cutanéo-muqueux hémorragiques peuvent apparaître spontanément (purpura pétéchial ou ecchymotique, bulle hémorragique, gingivorragie, épistaxis bilatérale...) [3,8-11]. Les complications hémorragiques viscérales (cérébroméningées, digestives) sont plus rares. Elles sont presque toujours précédées par un syndrome hémorragique cutanéomuqueux [3,10]. Elles ne se produisent habituellement que lorsque le taux de plaquettes est inférieur à 10 G. $\mathrm{I}^{-1}[3]$.

L'apparition d'un purpura chez un patient VIH positif avec thrombocytopénie sévère $\left(<20 \mathrm{G}^{-l^{-1}}\right)$ s'inscrit le plus souvent dans le cadre d'un purpura thrombopénique immunologique (PTI) [8-11]. Son diagnostic repose sur les éléments suivants: thrombocytopénie isolée sans anomalie des autres lignées, myélogramme montrant une moelle osseuse normale et riche en mégacaryocytes, présence d'autoanticorps antiplaquettes dans le sérum et/ou à la surface des plaquettes, élimination d'une cause médicamenteuse [3].

L'existence d'une thrombocytopénie sévère

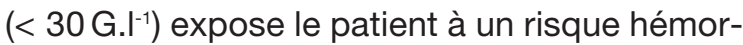
ragique dans le cadre d'une intervention chirurgicale [11,12].

L'utilisation d'immunoglobulines humaines polyvalentes par voie intraveineuse (IgIV) dans le cadre d'un protocole d'hémostase lors d'une intervention de chirurgie buccale chez un patient $\mathrm{VIH}$ positif est présentée.

Les différentes thérapeutiques dans la prise en charge des thrombocytopénies immunologiques liées à l'infection VIH seront ensuite discutées. 


\section{OBSERVATION}

Un homme, âgé de 40 ans, est adressé en avril 2000 par le Service de Médecine Interne et Maladies Infectieuses pour des douleurs localisées au maxillaire gauche. L'interrogatoire médical révèle que le patient est infecté par le VIH de type 1 depuis mai 1990. Le mode de contamination est hétérosexuel. II n'existe pas de co-infection par le virus de l'hépatite $C$. Sont survenues : une leucoplasie orale chevelue en 1992, et une candidose buccale en 1995. Début 1996, un taux de CD4+ inférieur à $100 / \mathrm{mm}^{3}$ marque le passage au stade SIDA. Un PTI est diagnostiqué en 1999 : numéra-

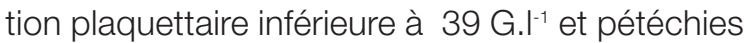
des membres inférieurs. L'origine périphérique et immunologique de cette thrombocytopénie est affirmée par le dosage plasmatique d'autoanticorps antiplaquettes. Par ailleurs le myélogramme est normal. Les sérologies des différents agents viraux autres que le $\mathrm{VIH}$ (cytomégalovirus, virus d'Epstein-Barr, virus de l'hépatite B et C) classiquement responsables de PTI sont négatives. II s'agit dans ce cas d'un PTI directement lié à l'infection $\mathrm{VIH}$. Le traitement antirétroviral repose sur l'association de deux inhibiteurs de la transcriptase reverse du VIH (stavudine, lamivudine) et de deux antiprotéases (ritonavir, saquinavir). L'examen clinique exobuccal est normal. L'examen endobuccal objective, sur le palais mou, de multiples macules hémorragiques, punctiformes, indolores, regroupées, qui ne s'effacent pas à la vitropres-

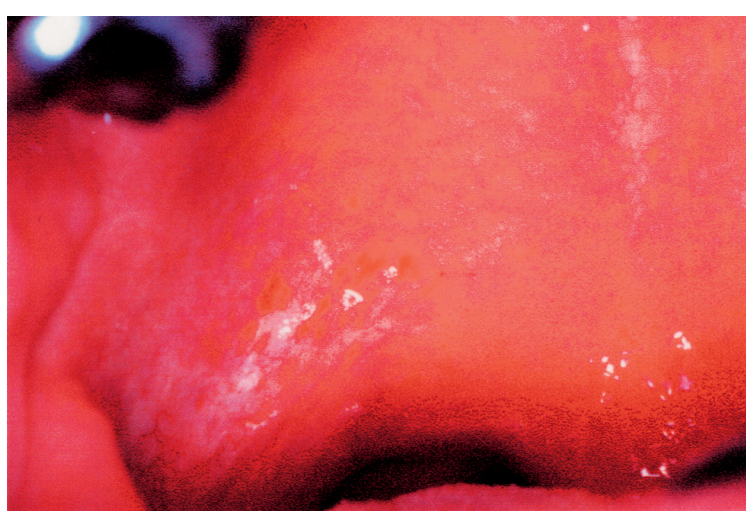

Figure 1: Vue clinique : pétéchies faisant évoquer un purpura.

Clinical view: purpura. sion. Le diagnostic positif d'un purpura sous forme de pétéchies est rapidement posé (Fig. 1). D'autre part, une parodontite apicale aiguë sur la 28 est diagnostiqué ainsi que d'importantes lésions carieuses sur 24 et 26. L'hygiène bucco-dentaire est insuffisante. Aucune gingivorragie spontanée n'est observée. Des clichés rétroalvéolaires ainsi qu'un orthopantomogramme (Fig. 2) confirment les données de l'examen clinique endobuccal. Devant ce tableau clinique, il est décidé de réaliser un détartrage et d'extraire 24, 26 et 28. Un bilan biologique préopératoire est prescrit ; il a pour but d'évaluer le risque de complications infectieuses et hémorragiques inhérentes à l'acte chirurgical programmé. La prise en charge thérapeutique de la parodontite apicale aiguë a consisté en un drainage canalaire associé à une prescription antibiotique (amoxicilline 1,5 g.j. ${ }^{-1}+$ métronidazole 1,5 g.j j $^{-1}$ pendant 10 jours). Le bilan préopératoire met en évidence une thrombocytopénie à $8 \mathrm{G} . \mathrm{ll}^{-1}$, un temps de saignement par méthode Ivy (TS - Ivy) supérieur à 10 minutes, un TP de $93 \%$, un TCA à 12,7 secondes, un taux de

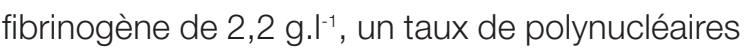
neutrophiles à $4,3 \mathrm{G} \mathrm{I}^{-1}$, un taux de $\mathrm{CD}^{+}$à $197 / \mathrm{mm}^{3}$ et une charge virale à 770 copies $/ \mathrm{ml}$. L'ensemble de ces résultats biologiques confirme l'existence d'une thrombocytopénie sévère isolée et l'absence de neutropénie. Le risque de complications hémorragiques est majeur ; en revanche le risque infectieux est considéré comme faible. Un protocole d'hémostase est défini avec le

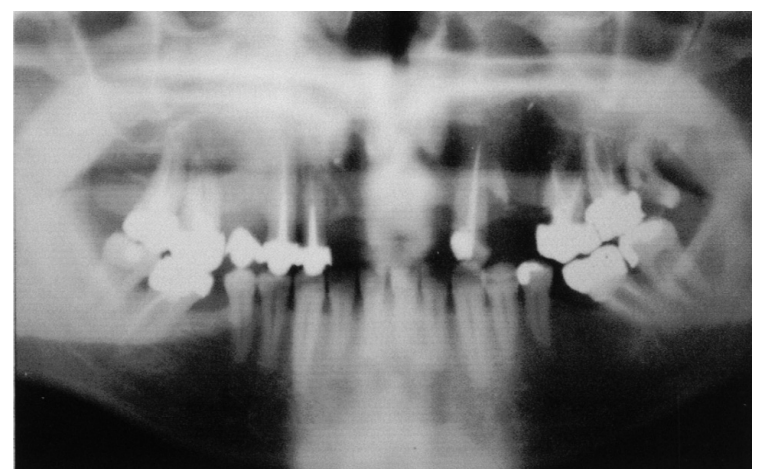

Figure 2 : Examen radiographique : cliché orthopantomographique montrant l'image de lésions carieuses au niveau de 24, 26 et 28.

Radiographic examination: Orthopantogram showing dental caries on 24, 26 and 28. \begin{tabular}{l}
$\begin{array}{l}\text { médecine } \\
\text { buccale } \\
\text { chirurgie } \\
\text { buccale }\end{array}$ \\
\hline VOL. 10, N N 2 \\
2004
\end{tabular}

page 73 
médecine

buccale

chirurgie

buccale

VOL. $10, \mathrm{~N}^{\circ} 2$ 2004

page 74
Service de Médecine Interne et Maladies Infectieuses : hospitalisation du patient, à J1 perfusion lente d'IgIV (Tégéline $®)$ à la posologie de $1 \mathrm{~g} \cdot \mathrm{kg}^{-1} \cdot \mathrm{j}^{-1}$, à J3 bilan biologique préopératoire et avulsions dentaires avec hémostase locale et surveillance médicale. Le bilan d'hémostase à J3 objective une remontée des plaquettes à un taux de $68 \mathrm{G}^{-\mathrm{I}^{-1}}$ et un TS - Ivy à 7 min 30. Les avulsions dentaires sont réalisées sous anesthésie locale par infiltration d'articaïne adrénalinée à 1/200.000 . L'hémostase locale est assurée par l'application d'une colle de fibrine (Tissucol®), la réalisation de sutures hermétiques, la pose d'une prothèse amovible partielle ajustée et rebasée avec une résine acrylique à prise retard (Viscogel囚). Après $48 \mathrm{~h}$ de surveillance, l'absence de saignement autorise la sortie du patient. Le patient est revu à J10 pour contrôle et dépose des sutures.

En 2001, des soins dentaires comprenant, détartrage, avulsions de 13, 27 et 37, sont réalisés selon le même protocole opératoire. Les IgIV permettent de faire remonter transitoirement le taux de pla-

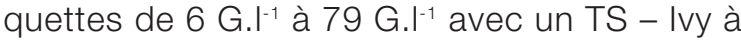
10 min 30. Lors de cette deuxième intervention chirurgicale, comme pour la première, aucune hémorragie per ou postopératoire n'est observée.

\section{COMMENTAIRES}

De très nombreuses anomalies acquises de l'hémostase ont été rapportées au cours de l'infection VIH. Les thrombocytopénies sont parmi les premières anomalies biologiques à avoir été observées au cours de l'infection VIH. En effet, dès 1982, des cas de thrombocytopénies sévères (<20 G. I $\left.^{-1}\right)$ sont rapportés chez des patients homosexuels américains par Morris et coll [8]. Une thrombocytopénie expose en principe à un risque de complication hémorragique en cas de geste médical invasif. Ce risque est considéré comme majeur lorsque en dehors de toute autre anomalie de l'hémostase, le taux de plaquettes

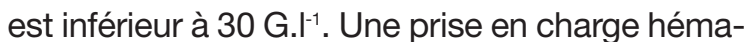
tologique est alors indispensable $[6,11,13]$. Le traitement classique des thrombocytopénies sévères ou du PTI repose principalement sur la corticothérapie, et en cas d'échec sur la splénectomie. D'autres traitements ont également été proposés : le danazol, l'interféron a, les immunoglobulines anti-D, certains immunosuppresseurs (azathioprine, cyclophosphamide) et les alcaloïdes de pervenche (vincristine, vinblastine) [11,13,14]. Ces traitements ne sont indiqués que chez les patients résistant aux corticoïdes. Leur efficacité est aléatoire, de plus ils n'ont été utilisés que sur un nombre trop faible de patients pour pouvoir juger de leur intérêt. En cas d'infection $\mathrm{VIH}$, des traitements plus spécifiques sont utilisés comme I'AZT et la dapsone [2,5,7]. L'AZT et la dapsone présentent une efficacité partielle avec un taux de réponse correcte (numération plaquettaire

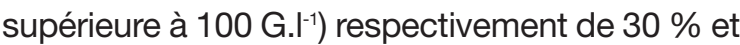
$6 \%[2,5]$.

Une autre thérapeutique est la splénectomie. Bien qu'étant le traitement le plus efficace à long terme chez les patients infectés par le VIH [2,11], la splénectomie expose à des complications infectieuses sévères, notamment à pneumocoques, à hémophilus et à méningocoques ; ce malgré une vaccination préventive $[15,16]$.

Indépendamment du traitement d'entretien, lorsqu'un taux de plaquettes efficace est rapidement exigé, en particulier en cas de préparation à une intervention chirurgicale, trois options thérapeutiques sont classiquement proposées : les concentrés de plaquettes, les corticoïdes ou les IgIV [2,5,14].

En cas de thrombocytopénie d'origine immunologique ou de PTI, les transfusions de plaquettes sont illogiques et coûteuses en dehors de la survenue d'une complication hémorragique per ou postopératoire. La destinée des plaquettes transfusées est identique à celles des plaquettes du patient et l'hyperdestruction en est aussi rapide [2] Les corticoïdes peuvent également être proposés. En effet, ils inhibent rapidement l'activité phagocytaire des macrophages spléniques et diminuent la synthèse des autoanticorps antiplaquettes par les plasmocytes. Plusieurs schémas posologiques ont été étudiés. La prednisolone, à la dose de 1 à 2 mg.kg-1.j-1 par voie orale pendant deux à trois semaines permet une remontée des plaquettes. Le taux de plaquettes se normalise entre 8 et 21 jours [14]. Des doses plus élevées de prednisolone permettent d'obtenir un délai d'action plus court. Une dose 
de 4 à $8 \mathrm{mg} \mathrm{kg}^{-1} \cdot \mathrm{j}^{-1}$ par voie orale pendant 1 semaine ou des bolus intraveineux de méthylprednisolone de 10 à $50 \mathrm{mg} \cdot \mathrm{kg}^{-1}$ pendant 3 jours permettent une remontée des plaquettes en 2 à 3 jours [14]. Malgré leur efficacité, les corticoïdes n'ont guère leur place dans la prise en charge d'une thrombocytopénie sévère chez le patient infecté par le VIH. Pour de nombreux auteurs [2,5], ils accroissent l'immunodépression et le risque de complications infectieuses notamment celles dues aux virus herpétiques humains et au Candida.

L'utilisation des IgIV dans le traitement des PTI est connue depuis 1981 [17-19]. Les IgIV sont des concentrés d'lgG humaines polyvalentes purifiés, contenant toutes les sous-classes d'lgG du plasma humain normal, et quelques traces d'lgM et d'IgA. Les IgIV sont obtenues à partir d'un mélange de plasmas de plus de 1000 donneurs sains [18]. En France, il existe actuellement 3 préparations commerciales d'IgIV : Tégéline $₫$, Sandoglobuline $\AA$ et Endobuline $\AA$. Leur mode d'action reste controversé mais plusieurs mécanismes peuvent s'associer : blocage direct des récepteurs Fc des macrophages, inhibition de la production et/ou neutralisation des autoanticorps antiplaquettes par modulation des fonctions régulatrices des lymphocytes $T$ [18]. Le schéma posologique actuel recommande une dose unique de $1 \mathrm{~g} . \mathrm{kg}^{-1}$ en perfusion lente [14,17]. La réponse aux IgIV est rapide : elle est effective en moins de 48 heures. $75 \%$ des malades traités sont répondeurs et on observe dès le $3^{\mathrm{e}}$ jour, une normalisation du taux de plaquettes. En revanche, leur durée d'action est limitée dans le temps ; néanmoins elle couvre largement la période per et postopératoire immédiate. On observe un retour à la valeur initiale généralement en 3 à 4 semaines [14,17].
Les IgIV sont relativement bien tolérées. Des effets indésirables bénins rapportés (céphalées, nausées) sont surtout liés à l'hypertonicité de la solution IgIV et s'amendent en ralentissant le débit de perfusion. Comme tout médicament dérivé du sang, malgré des conditions maximales de sécurité virale, il est impossible d'éliminer tout risque de transmission virale ou d'agents non conventionnels de type prion.

L'administration d'IgIV doit dans tous les cas s'associer à une hémostase locale adaptée. Les moyens d'hémostase locale regroupent l'utilisation de matériaux hémostatiques résorbables, de colle de fibrine et de sutures résorbables. Bartholomew et coll [20] rapportent l'efficacité des antifibrinolytiques administrés conjointement en application locale et en perfusion intraveineuse dans la prévention du risque hémorragique lors d'extractions dentaires.

Enfin, une hospitalisation est obligatoire afin d'assurer une surveillance postopératoire rigoureuse.

Ce cas clinique illustre la nécessité d'un examen biologique orienté avant une intervention chirurgicale chez le patient immunodéprimé, notamment en cas d'infection VIH. En cas de thrombocytopénie sévère $\left(<20 \mathrm{G} . \mathrm{I}^{-1}\right)$, le risque hémorragique peut être contrôlé par une hémostase locale associée à la prise d'IgIV. Une surveillance postopératoire dans le cadre d'une hospitaliation est recommandée. Les IgIV représentent le traitement médical des thrombocytopénies d'origine immunologique le plus efficace en terme de fréquence, d'intensité et de rapidité de réponse. En revanche, leur coût et leur effet transitoire limitent leur indication à des situations cliniques précises (intervention chirurgicale, syndrome hémorragique).

\section{RÉFÉRENCES}

1 - FAUCI AS, LANE HC. Infection par le virus de l'immunodéficience humaine $(\mathrm{VIH})$ : sida et maladies associés (pp 1566-1618). In : Harrison : Principes de Médecine Interne. $15^{\circ}$ ed, Flammarion- Médecine-Sciences, Paris 2002.
2 - Fezoui h, Garnier G, Taillan B, Cassuto Jp, Pesce A. Anomalies de l'hémostase et infection par le virus de l'immunodéficience humaine. Rev Méd Interne 1996 ; $17: 738-45$. médecine

buccale chirurgie buccale

VOL. $10, \mathrm{~N}^{\circ} 2$ 2004

page 75 
3 - Godeau B, BierLing P. Thrombopénie. Orientations diagnostiques. Rev Prat $1997 ; 47$ : 1695-701.

4 - Bettaleb A, Fromont P, Louache F. Presence of crossreactive antibody between human immunodeficiency virus (HIV) and platelet glycoproteins by HIV-related immune thrombocytopenic purpura. Blood 1992; 80 : 162-9.

5 - OKSENSENDLER E. Thrombopénies liées aux sida. Sang Thrombose Vaisseaux 1999; $11: 65-9$.

6 - PATTON LL. Hematologic abnormalities among HIV-infected patients. Associations of significance for dentistery. Oral Surg Oral Med Oral Pathol Oral Radiol Endod $1999 ; 88: 561-7$

7 - Toulon P. Hémostase et infection par le virus de l'immunodéficience humaine $(\mathrm{VIH})$. Ann Biol Clin 1998. 56: 153-60.

8 - Morris L, Distenfeld A, Amorosi E, Karpatkin S. Autoimmune thrombocytopenic purpura in homosexual men. Ann Intern Med 1982 ; 96 : 714-7.

9 - Maron G, Helmy E, Bays R. Immune thrombocytopenia in AIDS patients: case report. J Oral Maxillofac Surg $1989 ; 47: 1093-5$.

10 - LEVINE JD, Groopman JE. Hemostatic complications of HIV infection (pp1027-37). In : Thrombosis and hemorrhage. J. Loscalzo, Al Schafer. ed, Blackwell scientific Press, Boston, 1993.

11 - SeBaHOUN G. Purpura thrombopénique auto-immun (pp 181-8). In : Hématologie clinique et biologique. G. Sebahoun. ed, Arnette Initiatives Santé, Paris 1998.

12 - BouabDallah R, GastaUt JA. Transfusions plaquettaires chez le thrombopénique (pp 353-5). In : Hématologie clinique et biologique. G. Sebahoun. ed, Arnette Initiatives Santé, Paris 1998.
13 - Henderson JH, Bergman S, Salama A, Kotervas G. Management of the oral and maxillofacial surgery patient with thrombocytopenia. J Oral Maxillofac Surg 2001; $59: 421-7$.

14 - Macro M, Boutard P, Leporrier M. Purpura thrombopénique auto-immun. Modalités thérapeutiques. Presse Med $1997 ; 26: 439-43$.

15 - Fuzibet JG, Sanderson F, Gratecos N, Talllan B, BENCHIMOL D, PESCE A, DuJARDIN P. Effets à long terme de la splénectomie pour purpura thrombopénique immunologique lié au VIH. Presse Med 1992 ; 21 : 1534.

16 - SLeth JC, Senegas F, Gaday I. Echec de la vaccination anti-pneumococcique chez une patiente splénectomisée porteuse du VIH. Presse Med $1998 ; 27: 68$.

17 - GODEAU B, BierLING Ph. Immunoglobulines intraveineuses et purpura thrombopénique idiopathique : modalités thérapeutiques. Sang Thromb Vaiss 1999 ; 11: $60-4$

18 - Mouthon L, Nicoletti A, Lacroix-desmazes, KaVRI SV, KAZATCHKINE MD. Mécanismes d'action des immunoglobulines intraveineuses (IgIV). Sang Thromb Vaiss $1999 ; 11: 52-9$.

19 - Roussel LJ, Moisant L, Delhotellerie C, Merot S, PESCE A. Purpura thrombopénique auto-immun et syndromes apparentés au SIDA. Préparation à la chirurgie par les immunoglobulines. Ann Fr Anesth Reanim 1987; $6: 356-8$.

20 - Bartholomew JR, Salgia R, BelL WR. Control of bleeding in patients with immune and nonimmune thrombocytopenia with aminocaproic acid. Arch intern Med 1989 ; 149 : 1959-61. 